\title{
Middle meningeal artery embolization treatment of nonacute subdural hematomas in the elderly: a multiinstitutional experience of 151 cases
}

\author{
Evan Joyce, MD, MS, ${ }^{1}$ Michael T. Bounajem, MD, ${ }^{1}$ Jonathan Scoville, MD, ${ }^{1}$ Ajith J. Thomas, MD, ${ }^{2}$ \\ Christopher S. Ogilvy, MD, ${ }^{2}$ Howard A. Riina, MD, ${ }^{3}$ Omar Tanweer, MD, ${ }^{3}$ Elad I. Levy, MD, MBA, ${ }^{4}$ \\ Alejandro M. Spiotta, MD, ${ }^{5}$ Bradley A. Gross, MD, ${ }^{6}$ Brian T. Jankowitz, MD, ${ }^{7}$ \\ C. Michael Cawley, MD, ${ }^{8}$ Alexander A. Khalessi, MD, ${ }^{9}$ Aditya S. Pandey, MD, ${ }^{10}$ \\ Andrew J. Ringer, MD, ${ }^{11}$ Ricardo Hanel, MD, PhD, ${ }^{12}$ Rafael A. Ortiz, MD, ${ }^{13}$ David Langer, MD,13 \\ Michael R. Levitt, MD, ${ }^{14}$ Mandy Binning, MD, ${ }^{15}$ Philipp Taussky, MD, ${ }^{1}$ Peter Kan, MD, ${ }^{16}$ \\ and Ramesh Grandhi, MD1
}

${ }^{1}$ Department of Neurosurgery, Clinical Neuroscience Center, University of Utah, Salt Lake City, Utah; ${ }^{2}$ Neurosurgical Service, Beth Israel Deaconess Medical Center, Harvard Medical School, Boston, Massachusetts; ${ }^{3}$ Department of Neurosurgery, NYU Langone Medical Center, New York, New York; ${ }^{4}$ Department of Neurosurgery, University at Buffalo, State University of New York, Buffalo, New York; 5 Department of Neurosurgery, Medical College of South Carolina, Charleston, South Carolina; ${ }^{6}$ Department of Neurological Surgery, University of Pittsburgh Medical Center, Pittsburgh, Pennsylvania; ${ }^{7}$ Cooper Neuroscience Institute, Camden, New Jersey; ${ }^{8}$ Department of Neurosurgery, Emory University, Atlanta, Georgia; ${ }^{9}$ Department of Neurosurgery, University of California, San Diego, La Jolla, California; ${ }^{10}$ Department of Neurosurgery, University of Michigan, Ann Arbor,

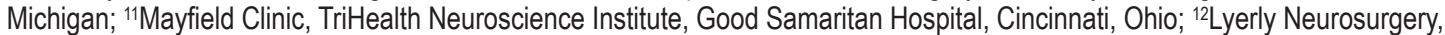
Baptist Neurological Institute, Jacksonville, Florida; ${ }^{13}$ Department of Neurosurgery, Lenox Hill Hospital, New York, New York; ${ }^{14}$ Department of Neurological Surgery, University of Washington, Seattle, Washington; ${ }^{15}$ Department of Neurosurgery, Drexel University College of Medicine, Philadelphia, Pennsylvania; and ${ }^{16}$ Department of Neurosurgery, Baylor College of Medicine, Houston, Texas

OBJECTIVE The incidence of already common chronic subdural hematomas (CSDHs) and other nonacute subdural hematomas (NASHs) in the elderly is expected to rise as the population ages over the coming decades. Surgical management is associated with recurrence and exposes elderly patients to perioperative and operative risks. Middle meningeal artery (MMA) embolization offers the potential for a minimally invasive, less morbid treatment in this age group. The clinical and radiographic outcomes after MMA embolization treatment for NASHs have not been adequately described in elderly patients. In this paper, the authors describe the clinical and radiographic outcomes after 151 cases of MMA embolization for NASHs among 121 elderly patients.

METHODS In a retrospective review of a prospectively maintained database across 15 US academic centers, the authors identified patients aged $\geq 65$ years who underwent MMA embolization for the treatment of NASHs between November 2017 and February 2020. Patient demographics, comorbidities, clinical and radiographic factors, treatment factors, and clinical outcomes were abstracted. Subgroup analysis was performed comparing elderly (age 65-79 years) and advanced elderly (age $>80$ years) patients.

RESULTS MMA embolization was successfully performed in $98 \%$ of NASHs (in 148 of 151 cases) in 121 patients. Seventy elderly patients underwent 87 embolization procedures, and 51 advanced elderly patients underwent 64 embolization procedures. Elderly and advanced elderly patients had similar rates of embolization for upfront (46\% vs 61\%), recurrent (39\% vs 33\%), and prophylactic (i.e., with concomitant surgical intervention; $15 \%$ vs $6 \%$ ) NASH treatment. Transfemoral access was used in most patients, and the procedure time was approximately 1 hour in both groups. Particle embolization with supplemental coils was most common, used in $51 \%$ (44/87) and $44 \%$ (28/64) of attempts for the elderly and advanced elderly groups, respectively. NASH thickness decreased significantly from initial thickness

ABBREVIATIONS CSDH = chronic subdural hematoma; $\mathrm{MMA}=$ middle meningeal artery; $\mathrm{NASH}=$ nonacute subdural hematoma .

SUBMITTED June 1, 2020. ACCEPTED July 17, 2020.

INCLUDE WHEN CITING DOI: 10.3171/2020.7.FOCUS20518. 
to 6 weeks, with additional decrease in thickness observed in both groups at 90 days. At longest follow-up, the treated NASHs had stabilized or improved in $91 \%$ and $98 \%$ of the elderly and advanced elderly groups, respectively, with $>50 \%$ improvement seen in $>60 \%$ of patients for each group. Surgical rescue was necessary in $4.6 \%$ and $7.8 \%$ of cases, and the overall mortality was $8.6 \%$ and $3.9 \%$ for elderly and advanced elderly patients, respectively.

CONCLUSIONS MMA embolization can be used safely and effectively as an alternative or adjunctive minimally invasive treatment for NASHs in elderly and advanced elderly patients.

https://thejns.org/doi/abs/10.3171/2020.7.FOCUS20518

KEYWORDS elderly; nonacute subdural hematoma; chronic subdural hematoma; middle meningeal artery; endovascular embolization

$\mathrm{N}$ ONACUTE subdural hematomas (NASHs) include subacute, acute-on-chronic, and chronic subdural hematomas (CSDHs) and disproportionally affect the elderly. As such, NASHs have become an ever more prevalent diagnosis, rising in concert with the aging population. ${ }^{1}$ The estimated incidence of CSDHs in the general population is projected to nearly double from 10.35 to 17.6 per 100,000 over the next decade, likely making it the most commonly treated cranial neurosurgical condition in adults by $2030 .^{2}$ The projected increase in both incidence and prevalence reflects the aging population, with approximately $20 \%-25 \%$ of the population expected to be older than 65 years by the turn of the next decade. ${ }^{3}$ Furthermore, the incidence of NASHs is dependent on age, and most cases occur in patients older than 65 years, with incidences estimated as high as 80.1 per 100,000 in those older than 65 years and 127.1 per 100,000 in those older than 80 years. ${ }^{4,5}$ Diffuse cerebral atrophy, more frequent use of antiplatelet and anticoagulant medications, and increased frequency of minor trauma such as falls in the elderly patient population are all believed to be important factors contributing to NASH development. ${ }^{1,6}$

There is still no definitive consensus on the most appropriate treatment for NASHs, particularly in the elderly, and, as such, there remains wide variation in practice, ranging from conservative management to operative intervention. ${ }^{78}$ Medical management of mildly symptomatic CSDHs has thus far also failed to improve outcomes. For example, the randomized, placebo-controlled ATOCH trial comparing an 8-week course of atorvastatin with placebo for mildly symptomatic CSDH reported neurological improvement in only $45 \%$ of patients receiving atorvastatin compared with $28 \%$ of the placebo group, and surgical rescue for neurological deterioration was needed in $11.2 \%$ and $23.5 \%$ of the atorvastatin and placebo groups, respectively. ${ }^{9}$ Surgical management with twist-drill craniostomy, burr hole craniotomy, or standard craniotomy under general anesthesia remains the most common method for drainage for both asymptomatic and symptomatic subdural hematomas. Unfortunately, postoperative recurrence remains common, with estimates as high as $30 \%{ }^{10} \mathrm{An}$ other challenge complicating NASH treatment in the surgical perioperative period is deciding when and for how long antiplatelet or anticoagulation medications should be held. ${ }^{11}$ The decision to operatively treat subdural hematomas in the elderly remains complicated: they have more medical complexity, are at increased risk of cardiac complications from general anesthesia, are more susceptible to postoperative infections, and are at risk of deconditioning during prolonged postoperative bed rest. ${ }^{712-14}$
Middle meningeal artery (MMA) embolization is a newly recognized technique used as an alternative or adjunctive minimally invasive treatment for NASHs. Early studies have uniformly demonstrated favorable outcomes with better efficacy and safety compared with conventional surgical evacuation. ${ }^{15,16}$ In addition to decreased procedure-related morbidity, the benefits of MMA embolization are thought to be derived from improved targeting of the underlying pathophysiology of the subdural hematomas (i.e., devascularizing the immature capillary network supplying the fibrocellular neomembranes formed after subdural blood is reorganized), which is thought to decrease repeated cycles of subdural rebleeding, recurrence, and expansion over time. ${ }^{17}$ Recent reports have primarily explored the use of MMA embolization for treating CSDHs in patients who are unable to stop anticoagulant therapy ${ }^{18}$ or patients with recurrent hematomas, ${ }^{19-22}$ however, aside from a few, larger single-center cohorts, ${ }^{23,24}$ most reports comprise small case series. MMA embolization has yet to be adequately studied among the elderly. The purpose of this study was to analyze a cohort of elderly patients who underwent MMA embolization for NASH treatment at 15 academic centers across the US and describe the clinical and radiographic outcomes in this population.

\section{Methods}

We retrospectively reviewed a prospectively collected database of patients who underwent MMA embolization for NASHs between November 2017 and February 2020 across 15 US academic centers. Each center obtained appropriate local institutional review board approval before data collection.

The database was queried for patients aged 65 years and older who were further divided into two groups: age 65-79 years (elderly) and age $\geq 80$ years (advanced elderly). Patient charts were reviewed for demographics, clinical data, and radiographic data, including age, sex, race, comorbidities, prior NASH, prior NASH evacuation, use of antiplatelet or anticoagulant medications, midline shift, laterality of hematoma, maximum hematoma width on axial imaging, and presence of membranes. Treatment data were also recorded, including embolization success, NASH treatment indication (upfront, recurrent, or prophylactic with concurrent surgical intervention), access location (transfemoral vs transradial), type of anesthesia (general anesthesia vs moderate sedation), procedure duration, and type of embolysate used (coils, liquid embolic, particles, or a combination). The maximum width of the subdural hematoma was measured initially, at 6 weeks, and 
at 90 days and was also categorized as worse, improved, $>50 \%$ reduction, or $100 \%$ reduction at the latest recorded follow-up. The need for retreatment, adverse events, and mortality were assessed at the latest recorded follow-up.

Statistical analysis in the form of Student t-tests was conducted to compare midline shift and maximum subdural width between the two age groups, as well as to compare preprocedural width and width at 6-week and 90-day follow-ups. Welch's t-test was used to compare the average age between the groups. Chi-square analysis was conducted to compare preprocedure antiplatelet/anticoagulant use, sex, indication for procedure, outcome at latest follow-up, and mortality between the two age groups.

\section{Results}

In total, MMA embolization was attempted on 151 NASHs in 121 patients (30 bilateral procedures). Seventy elderly patients underwent a total of 87 embolization attempts (17 bilateral), and 51 advanced elderly patients underwent a total of 64 embolization attempts (13 bilateral) (Table 1). The average age for the elderly and advanced elderly patients differed significantly at 72.3 and 85.2 years old, respectively $(\mathrm{p}<0.005)$. Both groups were predominantly white and male. Comorbid conditions were similar, with hypertension being the most common, followed closely by hyperlipidemia, coronary artery disease, and diabetes mellitus. Antiplatelet or anticoagulant agents were more common in the advanced elderly than in the elderly patients $(69 \%$ vs $49 \%$; $p=0.03)$. The initial maximum subdural width was significantly greater in the advanced elderly group ( 16.2 vs $14.5 \mathrm{~mm} ; \mathrm{p}=0.04$ ), whereas midline shift was significantly greater in the elderly group ( 2.0 vs $0.82 \mathrm{~mm} ; \mathrm{p}=0.006$ ). Subdural membranes were present in three-quarters of all NASHs (Table 2).

Of the 151 MMA embolization attempts, 98\% (in 148 of 151 cases) were successful. The 3 cases with failed attempts occurred in the younger group (Table 3). NASH treatment was performed in three settings. Upfront treatment was performed in 46\% (40/87) and 61\% (39/64) of cases in the elderly and advanced elderly groups, respectively. In the elderly group, prior evacuation had been performed in 32 cases, primarily with either craniotomy $(50 \% ; 16 / 32)$ or burr holes $(47 \% ; 15 / 32)$. Similarly, in the advanced elderly group, prior evacuation had been performed in 21 cases, primarily with either craniotomy (29\%; $6 / 21)$ or burr holes $(52 \% ; 11 / 21)$. Thus, MMA embolization was performed as a rescue treatment in 37\% (32/87) and $33 \%(21 / 64)$ of cases in the elderly and advanced elderly groups, respectively. Finally, prophylactic treatment was performed in 15\% (13/87) and 6\% (4/64) of cases in the elderly and advanced elderly groups, respectively.

Transfemoral access was used more than $80 \%$ of the time for each group, and general anesthesia was used in about half of cases for both groups (Table 3). The average procedure duration was nearly identical between the groups at just over 1 hour. None of these were significantly different between the groups.

The choice of embolic agent was similar between the two groups, with the most common being particles plus coils $(51 \%$ in the elderly group and $44 \%$ in the advanced
TABLE 1. Patient characteristics of 121 patients who underwent MMA embolization for NASH treatment

\begin{tabular}{|c|c|c|c|}
\hline \multirow[b]{2}{*}{ Variable } & \multicolumn{2}{|c|}{ Age Group, Yrs } & \multirow[b]{2}{*}{$\mathrm{p}$ Value } \\
\hline & $65-79$ & $\geq 80$ & \\
\hline No. of patients & 70 & 51 & \\
\hline Male sex & $59(84)$ & $40(78)$ & 0.41 \\
\hline Mean age, yrs & $72 \pm 3.7$ & $85 \pm 4.4$ & $<0.005$ \\
\hline \multicolumn{4}{|l|}{ Race } \\
\hline Hispanic & $8(11)$ & $4(8)$ & \\
\hline White & $52(74)$ & $34(67)$ & \\
\hline African American & $4(6)$ & $5(10)$ & \\
\hline Asian & $1(1)$ & $2(4)$ & \\
\hline Native American & $1(1)$ & 0 & \\
\hline \multicolumn{4}{|l|}{ Comorbidities } \\
\hline HTN & $30(43)$ & $34(67)$ & \\
\hline HLD & $23(33)$ & $19(37)$ & \\
\hline DM & $14(20)$ & $9(18)$ & \\
\hline CAD & $10(14)$ & $15(29)$ & \\
\hline CVD & $8(11)$ & $4(8)$ & \\
\hline $\mathrm{TBI}$ & $2(3)$ & $2(4)$ & \\
\hline PVD & $1(1)$ & $3(6)$ & \\
\hline Factor VI deficiency & $1(1)$ & $0(0)$ & \\
\hline Antiphospholipid syndrome & $1(1)$ & $0(0)$ & \\
\hline $\begin{array}{l}\text { Antiplatelet or anticoagulation } \\
\text { medication use }\end{array}$ & $34(49)$ & $35(69)$ & 0.03 \\
\hline $81 \mathrm{mg}$ aspirin & $13(19)$ & $14(27)$ & \\
\hline $325 \mathrm{mg}$ aspirin & $1(1)$ & $3(6)$ & \\
\hline Clopidogrel & $6(9)$ & $2(4)$ & \\
\hline Warfarin & $11(16)$ & $6(12)$ & \\
\hline Heparin & $0(0)$ & $1(2)$ & \\
\hline Apixaban & $2(3)$ & $4(8)$ & \\
\hline Dabigatran & $0(0)$ & $1(2)$ & \\
\hline Rivaroxaban & $1(1)$ & $1(2)$ & \\
\hline
\end{tabular}

$C A D=$ coronary artery disease $; C V D=$ cerebrovascular disease; $D M=$ diabetes mellitus; HLD = hyperlipidemia; $H T N$ = hypertension; PVD = peripheral vascular disease; $\mathrm{TBI}=$ traumatic brain injury.

Values represent the number of patients (\%) unless stated otherwise. The mean value is presented as the mean $\pm S D$. Boldface type indicates statistical significance.

elderly group). Embosphere Microspheres (Merit Medical) were used in nearly two-thirds of the particle embolizations, most commonly in the 100 - to $300-\mu \mathrm{m}$ or $300-$ to $500-\mu \mathrm{m}$ range, while polyvinyl alcohol particles were used in the remaining one-third, usually in the 150- to $250-\mu \mathrm{m}$ range or 250- to 355- $\mu \mathrm{m}$ range (data not shown). Among liquid embolics, Onyx (18 or 34, Medtronic) was used in $90 \%$ of cases, while $N$-butyl cyanoacrylate (TRUFILL, J\&J Medical Devices) was used in the remaining cases (data not shown).

At the 6-week follow-up, the maximum subdural width was not significantly different between groups (10.9 vs $9.31 \mathrm{~mm}$ in the elderly and advanced elderly groups; $\mathrm{p}$ $=0.28$ ); however, the decrease observed was significant when compared with the preprocedural subdural thick- 
TABLE 2. Clinical and radiographic factors of 121 patients who underwent MMA embolization for NASH treatment

\begin{tabular}{lccc}
\hline \multirow{2}{*}{ Variable } & \multicolumn{2}{c}{ Age Group, Yrs } & \multirow{2}{*}{\begin{tabular}{c} 
V \\
\cline { 2 - 3 } Value
\end{tabular}} \\
\cline { 2 - 3 } Mean maximum hematoma width & $65-79$ & $\geq 80$ & \\
\hline Mean midline shift & $2.0 \pm 3.2$ & $0.82 \pm 2.2$ & $\mathbf{0 . 0 0 6}$ \\
\hline Left side & $46(53)$ & $36(56)$ & \\
\hline Membranes & $67(77)$ & $51(80)$ & 0.69 \\
\hline Prior NASH evacuation & 32 & 21 & 0.62 \\
\hline Craniotomy & $16(50)$ & $6(29)$ & \\
\hline Burr holes & $15(47)$ & $11(52)$ & \\
\hline SEPS & $1(3)$ & $4(19)$ & \\
\hline
\end{tabular}

SEPS = subdural evacuating port system.

Values represent the number of cases (\%) unless stated otherwise. Mean values are presented as the mean \pm SD. Boldface type indicates statistical significance.

ness $(\mathrm{p}=0.04$ and $\mathrm{p}<0.001$ for the elderly and advanced elderly groups) (Table 4). At the 90-day follow-up, a similar trend was observed, with NASH thickness similar between the groups (4.66 vs $6.13 \mathrm{~mm} ; \mathrm{p}=0.22$ ). Thickness was significantly decreased from the 6-week follow-up in the advanced elderly group $(p=0.002)$. At the longest follow-up of at least 6 weeks, the majority of patients in each group showed NASH improvement. Data were available for $92 \%$ (80/87) and 91\% (58/64) of procedures in the elderly and advanced elderly groups, respectively. NASH stability or improvement was observed in $91 \%(73 / 80)$ and 98\% (57/58) in the elderly and advanced elderly groups, respectively. Similarly, $>50 \%$ improvement was observed in the majority of elderly (61\%) and advanced elderly (64\%), while complete resolution was observed in $24 \%$ and $17 \%$, respectively, for the two groups (Fig. 1). Worsening of the NASHs was infrequently observed (in $8.8 \%$ and $1.7 \%$ of the elderly and advanced elderly groups, respectively).

Adverse events were rare, at 2.3\% (2/87) in the elderly group (one delayed seizure and one delayed infarction) and $1.6 \%(1 / 64)$ in the advanced elderly group (intermittent aphasia). The need for surgical rescue, defined by post-MMA embolization burr holes or craniotomy for worsening or persistent symptoms, did not differ between the two groups, with $4.6 \%(4 / 87)$ and $7.8 \%(5 / 64)$ in the elderly and advanced elderly groups, respectively. Mortality was not significantly different between the groups, at a rate of $8.6 \%(6 / 70)$ in the elderly group and 3.9\% (2/51) in the advanced elderly group $(\mathrm{p}=0.31)$. Three patient deaths $(2.5 \%)$ occurred within 24 hours of the MMA embolization procedure, with only 1 neurological death due to the patient developing a cerebellar hematoma, remote from the subdural hematoma. The remaining 5 patient deaths (4.1\%) occurred between 2 and 6 weeks after the procedure and were all related to progression of underlying neoplastic disease or withdrawal of care by family.

\section{Discussion}

In this study, we describe the clinical and radiographic outcomes in a robust series of 121 elderly patients under-
TABLE 3. Treatment characteristics of 121 patients who underwent MMA embolization for NASH treatment

\begin{tabular}{|c|c|c|c|}
\hline \multirow[b]{2}{*}{ Variable } & \multicolumn{2}{|c|}{ Age Group, Yrs } & \multirow[b]{2}{*}{$\mathrm{p}$ Value } \\
\hline & $65-79$ & $\geq 80$ & \\
\hline \multicolumn{4}{|l|}{ Total no. of treatments } \\
\hline Attempted & 87 & 64 & \\
\hline Completed & $84(97)$ & $64(100)$ & 0.13 \\
\hline \multicolumn{4}{|l|}{ Treatment type } \\
\hline Upfront & $40(46)$ & $39(61)$ & 0.07 \\
\hline Recurrent & $34(39)$ & $21(33)$ & 0.43 \\
\hline Prophylactic & $13(15)$ & $4(6)$ & 0.09 \\
\hline Access & & & 0.86 \\
\hline TRA & $14(16)$ & $11(17)$ & \\
\hline TFA & $73(84)$ & $53(83)$ & \\
\hline Anesthesia & & & 0.18 \\
\hline General anesthesia & $38(44)$ & $35(55)$ & \\
\hline Moderate sedation & $49(56)$ & $29(45)$ & \\
\hline Mean procedure duration, mins & $67.6 \pm 27.3$ & $66.2 \pm 30.6$ & 0.38 \\
\hline \multicolumn{4}{|l|}{ Embolic agent } \\
\hline Coils & $3(3)$ & $3(5)$ & \\
\hline Liquid & $18(21)$ & $12(19)$ & \\
\hline Particles & $20(23)$ & $18(28)$ & \\
\hline Liquid + coils & $1(1)$ & $1(2)$ & \\
\hline Particles + coils & $44(51)$ & $28(44)$ & \\
\hline Particles + liquid & $1(1)$ & $0(0)$ & \\
\hline Bilateral procedure & $15(20)$ & $13(20)$ & 0.91 \\
\hline
\end{tabular}

TFA $=$ transfemoral access; TRA $=$ transradial access

Values represent the number of cases (\%) unless stated otherwise. The mean value is presented as the mean $\pm \mathrm{SD}$.

going 151 embolization procedures to treat NASHs. To the best of our knowledge, this is the first such report of MMA embolization for subdural treatment in elderly patients. Overall, MMA embolization was highly efficacious in treating the vast majority of NASHs. Hematoma size either stabilized or improved at the longest follow-up in $91 \%$ and $98 \%$ in the elderly and advanced elderly groups, respectively, with surgical rescue needed in only $4.6 \%$ and $7.8 \%$ of cases for persistent or worsening symptoms. This represents an improvement compared with conventional surgical management, where retreatment rates are expected to exceed 20\%. ${ }^{24}$ Furthermore, there was $>50 \%$ subdural improvement in $>60 \%$ of patients in each group. Our subgroups were fairly equivalent in clinical, radiographic, and outcome measures, although the advanced elderly group was significantly more likely to be taking antiplatelet or anticoagulant medication and had greater initial NASH width with less initial midline shift. Upfront hematoma treatments were slightly more common in the advanced elderly group, but the difference did not reach statistical significance.

Our study provides additional evidence reinforcing the results from other recent large, single-center MMA embolization cohorts. ${ }^{15,23,24}$ In our patients, MMA embolization was both effective and safe in upfront subdural treatment, 
TABLE 4. Outcomes of 121 patients who underwent MMA embolization for NASH treatment

\begin{tabular}{|c|c|c|c|}
\hline \multirow[b]{2}{*}{ Variable } & \multicolumn{2}{|c|}{ Age Group, Yrs } & \multirow[b]{2}{*}{$\mathrm{p}$ Value } \\
\hline & $65-79$ & $\geq 80$ & \\
\hline \multicolumn{4}{|l|}{ Mean maximum width, mm } \\
\hline Initial & $14.5 \pm 6.0$ & $16.2 \pm 5.5$ & 0.04 \\
\hline No. of patients & 87 & 64 & \\
\hline 6-wk follow-up & $10.9 \pm 14.5^{*}$ & $9.3 \pm 8.4^{*}$ & 0.28 \\
\hline No. of patients & 55 & 38 & \\
\hline 90-day follow-up & $4.7 \pm 10.0$ & $6.1 \pm 4.6^{*}$ & 0.22 \\
\hline No. of patients & 42 & 34 & \\
\hline \multicolumn{4}{|l|}{ Longest follow-up } \\
\hline Data available & $80 / 87$ & $58 / 64$ & \\
\hline Worse & $7(8.8)$ & $1(1.7)$ & 0.08 \\
\hline Stable to improved & $73(91)$ & $57(98)$ & 0.08 \\
\hline$>50 \%$ improvement & $49(61)$ & $37(64)$ & 0.76 \\
\hline $100 \%$ improvement & $19(24)$ & $10(17)$ & 0.35 \\
\hline Adverse events & $2(2.3)$ & $1(1.6)$ & 0.75 \\
\hline Intermittent aphasia & 0 & 1 & \\
\hline Delayed seizure & 1 & 0 & \\
\hline Delayed infarction & 1 & 0 & \\
\hline Surgical rescue & $4(4.6)$ & $5(7.8)$ & 0.41 \\
\hline Mortality, no. of patients (\%) & $6(8.6)$ & $2(3.9)$ & 0.31 \\
\hline
\end{tabular}

Values represent the number of cases (\%) unless stated otherwise. Mean values are presented as the mean \pm SD. Boldface type indicates statistical significance.

* Significant difference from prior measurement.

in recurrent hematomas, and as a prophylactic therapy at the time of surgical intervention. In 2019, Link et al. ${ }^{23}$ reported their single-center experience of the first 60 cases of CSDHs treated with MMA embolization in 49 patients. Their cohort included 42 upfront, 8 recurrent, and 10 prophylactically treated subdural hematomas, and they reported comparable outcomes to those in our present study, with subdural stability or improvement in $91.1 \%$, an $8.9 \%$ recurrence rate, and no procedural complications. Interestingly, the average age of their cohort was younger (average age 69 years) than that of our subgroups, but antiplatelet or anticoagulation medication use was higher (80\%). Ban et al. ${ }^{24}$ also recently reported a large, single-center series of 72 consecutive patients prospectively identified and treated with MMA embolization for CSDHs. They included patients $\geq 20$ years of age and observed an average patient age of 69 years, as well as $40 \%$ taking antithrombotics. Enrollment criteria included CSDH thickness of at least $10 \mathrm{~mm}$, and the average subdural maximum width was slightly larger than that in our patient cohort at $19.6 \mathrm{~mm}$. MMA embolization was used alone (27/72) in asymptomatic patients or in combination with surgical hematoma evacuation $(45 / 72)$ in symptomatic patients. At the 6-month follow-up, there were no cases of surgical rescue for those treated with embolization alone and in only 1 (2.2\%) of 45 patients treated with embolization followed by surgery. Their cohort was compared with a historical control group of 469 patients with CSDH managed with
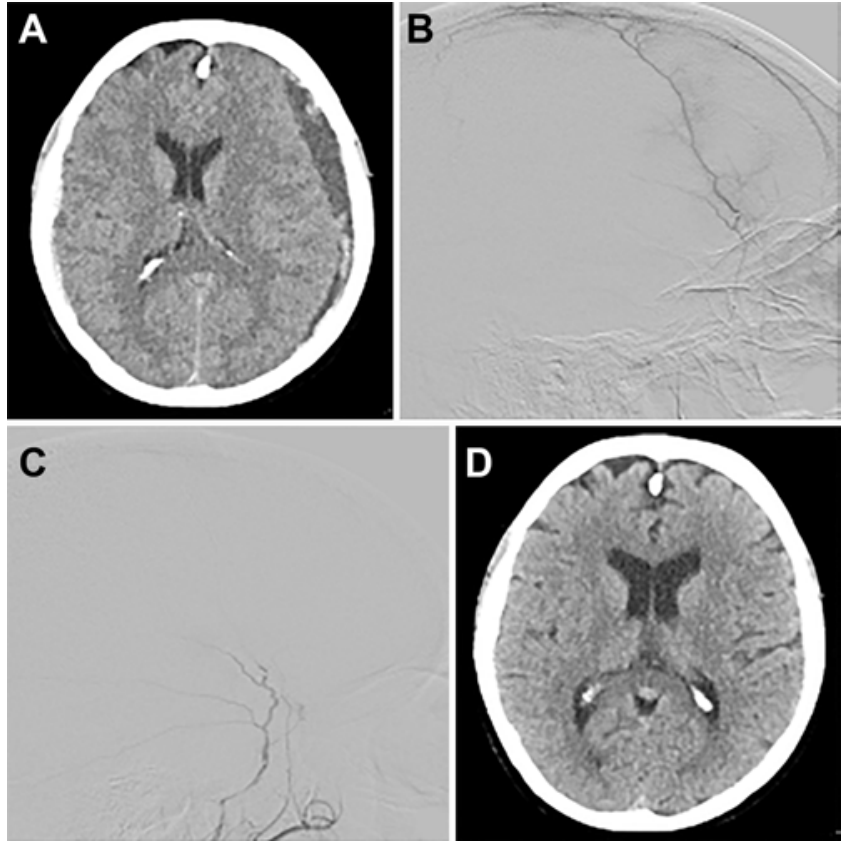

FIG. 1. Example of a left-sided NASH treated with MMA embolization. This 67 -year-old female patient on a regimen of coumadin for a recent pulmonary embolism in the setting of antiphospholipid syndrome presented with headache and a supratherapeutic international normalized ratio of 4.2. The hematoma measured $11.0 \mathrm{~mm}$ at presentation on an axial noncontrast CT scan (A). It was treated with embolization of the anterior and middle branches of the MMA using 100- to 300- $\mu \mathrm{m}$ Embosphere Microspheres (Merit Medical) (B and C; digital subtraction angiograms, lateral view). At the 90-day follow-up, the NASH had completely resolved as seen on the axial noncontrast CT scan (D).

conventional treatment (67 with conservative management and 402 with surgical evacuation), and they found significantly lower treatment failure rates as defined by residual hematoma thickness $>10 \mathrm{~mm}(1.4 \%$ vs $27.5 \% ; \mathrm{p}=0.001)$, less frequent surgical rescue (1.4\% vs $18.8 \% ; \mathrm{p}=0.005)$, and no difference in treatment complications ( $0 \%$ vs $4.3 \%$; $\mathrm{p}=0.182$ ). Recent systematic reviews have corroborated these excellent outcomes. ${ }^{16,25-27}$ The hematoma resolution rate was estimated to be as high as $96.8 \%,{ }^{27}$ with recurrence ranging from $2.1 \%$ to $4.1 \% 16,25,26$ and procedural complication rates from $0 \%$ to $2.1 \% .^{16,25-27}$

Surgery for CSDHs in the elderly carries unique challenges regarding effectiveness, recurrence rates, surgical complications, and medical perioperative complications. ${ }^{14,28-35}$ A 2018 review of adults older than 65 years $(2,831,274$ cases in the NSQIP registry and 1,952,305 Medicare beneficiaries) found that the rate of postoperative hematoma recurrence requiring repeat surgery was between 5\% and 10\%. ${ }^{36}$ For example, a Scandinavian population-based multicenter study of 1254 patients undergoing primary burr hole craniotomy found no statistically significant difference between surgical complications, perioperative morbidity, or perioperative mortality in patients older than 90 years versus those younger than 90 years..$^{29}$ Conversely, Krupa et al. ${ }^{32}$ found a dramatic difference in outcome when comparing burr hole craniotomy and standard craniotomy in the young (age $<40$ years) 
and elderly (age $>75$ years). Good outcome (Glasgow Outcome Scale score of 5) only occurred in $15.5 \%$ and $4.8 \%$ of their elderly patients treated with burr hole craniotomy and standard craniotomy, respectively, compared with a good outcome in approximately $70 \%$ of the younger group for each surgical method. Rohde et al. ${ }^{35}$ also found a higher surgical complication rate and overall mortality rate in elderly patients who underwent burr hole craniotomy for CSDH. Still others have reported unique perioperative risks that need to be monitored for in the elderly, such as a higher risk of postoperative acute subdural hematoma requiring treatment, ${ }^{34}$ higher risk of postoperative infection after craniotomy, ${ }^{12}$ higher risk of mortality, ${ }^{31}$ and decreased likelihood of a good functional outcome postoperatively. ${ }^{31,34}$ In addition to operative risks, the elderly are also at higher risk of perioperative medical complications. A retrospective review of 106 patients aged 60 years or older treated with burr hole craniotomy for CSDHs saw a higher frequency of cardiac complications, and thus longer hospitalization, in those receiving general anesthesia when compared with local anesthesia. ${ }^{14}$

Endovascular MMA embolization offers several advantages over conventional open surgery for subdural evacuation that make it a particularly attractive minimally invasive treatment option in elderly patients. Percutaneous transradial or transfemoral access are both generally well tolerated under local anesthesia with a low risk of complications. Most patients in our study underwent transfemoral access; however, transradial access may soon prove to be a superior approach because it has been shown to be associated with a lower risk of major bleeding and vascular complications, shorter time to ambulation, and, in some instances, an improved ability to navigate tortuous cerebrovascular anatomy. ${ }^{37,38}$ As such, MMA embolization procedures can often be done under moderate sedation or local anesthesia, thereby mitigating the risk of cardiac and respiratory complications from the stresses of endotracheal intubation and general anesthesia. Procedure times are also short; in the present study, the average duration was just over an hour in both subgroups. Postoperative bed rest is still practiced by clinicians as a way to allow brain reexpansion after subdural drain placement, but after MMA embolization, this is unnecessary as there is no subdural drain. Patients are typically ambulatory within a few hours after embolization, and in select asymptomatic patients, discharge to home may be appropriate after a short period of observation. Finally, infection risk is greatly reduced without the need for skin incision, craniotomy, and subdural drain placement.

A significant limitation of this study is that it is a nonrandomized, retrospective chart review of prospectively collected data and, as such, only demonstrates associations rather than causation. We also were unable to control for confounding variables, such as initial hematoma width, midline shift, and preprocedure antiplatelet or anticoagulation medication use. A unique strength of this cohort is the inclusion of 15 academic centers across the US. This helps increase the generalizability and external validity of our results; however, the wide variety of embolization agents used may make it challenging to draw meaningful conclusions as to which type of embolization is most ef- fective in elderly patients. To the best of our knowledge, this study presents the outcomes on the largest cohort of elderly patients to have undergone MMA embolization for treatment of NASH.

\section{Conclusions}

MMA embolization appears to be both a safe and efficacious minimally invasive treatment option for NASHs in the elderly.

\section{Acknowledgments}

We would like to acknowledge Kristin Kraus, MSc, for editorial assistance.

\section{References}

1. Yang W, Huang J. Chronic subdural hematoma: epidemiology and natural history. Neurosurg Clin N Am. 2017;28(2):205-210.

2. Balser D, Farooq S, Mehmood T, et al. Actual and projected incidence rates for chronic subdural hematomas in United States Veterans Administration and civilian populations. $J$ Neurosurg. 2015;123(5):1209-1215.

3. Vincent GK, Velkoff VA. The Next Four Decades: The Older Population in the United States: 2010 to 2050. Current Population Reports. US Census Bureau; 2010.

4. Mori K, Maeda M. Surgical treatment of chronic subdural hematoma in 500 consecutive cases: clinical characteristics, surgical outcome, complications, and recurrence rate. Neurol Med Chir (Tokyo). 2001;41(8):371-381.

5. Karibe H, Kameyama M, Kawase M, et al. Epidemiology of chronic subdural hematomas. Article in Japanese. No Shinkei Geka. 2011;39(12):1149-1153.

6. Juratli TA, Klein J, Schackert G. Chronic subdural hematoma in the elderly. Article in German. Chirurg. 2017;88(2): 131-135.

7. Shapey J, Glancz LJ, Brennan PM. Chronic subdural haematoma in the elderly: is it time for a new paradigm in management? Curr Geriatr Rep. 2016;5:71-77.

8. Mehta V, Harward SC, Sankey EW, et al. Evidence based diagnosis and management of chronic subdural hematoma: a review of the literature. J Clin Neurosci. 2018;50:7-15.

9. Jiang R, Zhao S, Wang R, et al. Safety and efficacy of atorvastatin for chronic subdural hematoma in chinese patients: a randomized clinical trial. JAMA Neurol. 2018;75(11):13381346.

10. Oh HJ, Lee KS, Shim JJ, et al. Postoperative course and recurrence of chronic subdural hematoma. J Korean Neurosurg Soc. 2010;48(6):518-523.

11. Scerrati A, Germanò A, Trevisi G, et al. Timing of low-dose aspirin discontinuation and the influence on clinical outcome of patients undergoing surgery for chronic subdural hematoma. World Neurosurg. 2019;129:e695-e699.

12. Champagne P-O, Brunette-Clement T, Bojanowski MW, et al. Safety of performing craniotomy in the elderly: the utility of co-morbidity indices. Interdiscip Neurosurg. 2018;14:97-101.

13. Uno M, Toi H, Hirai S. Chronic subdural hematoma in elderly patients: is this disease benign? Neurol Med Chir (Tokyo). 2017;57(8):402-409.

14. Kim S, Jung S, Won Y, et al. A comparative study of local versus general anesthesia for chronic subdural hematoma in elderly patients over 60 years. Korean J Neurotrauma. 2013; 9(2):47-51.

15. Fiorella D, Arthur AS. Middle meningeal artery embolization for the management of chronic subdural hematoma. J Neurointerv Surg. 2019;11(9):912-915.

16. Srivatsan A, Mohanty A, Nascimento FA, et al. Middle men- 
ingeal artery embolization for chronic subdural hematoma: meta-analysis and systematic review. World Neurosurg. 2019; 122:613-619.

17. Edlmann E, Giorgi-Coll S, Whitfield PC, et al. Pathophysiology of chronic subdural haematoma: inflammation, angiogenesis and implications for pharmacotherapy. J Neuroinflammation. 2017;14(1):108.

18. Hirai S, Ono J, Odaki M, et al. Embolization of the middle meningeal artery for refractory chronic subdural haematoma. usefulness for patients under anticoagulant therapy. Interv Neuroradiol. 2004;10(suppl 2):101-104.

19. Ishihara H, Ishihara S, Kohyama $S$, et al. Experience in endovascular treatment of recurrent chronic subdural hematoma. Interv Neuroradiol. 2007;13(suppl 1):141-144.

20. Kim E. Embolization therapy for refractory hemorrhage in patients with chronic subdural hematomas. World Neurosurg. 2017;101:520-527.

21. Mino M, Nishimura S, Hori E, et al. Efficacy of middle meningeal artery embolization in the treatment of refractory chronic subdural hematoma. Surg Neurol Int. 2010;1:78.

22. Tempaku A, Yamauchi S, Ikeda H, et al. Usefulness of interventional embolization of the middle meningeal artery for recurrent chronic subdural hematoma: five cases and a review of the literature. Interv Neuroradiol. 2015;21(3):366-371.

23. Link TW, Boddu S, Paine SM, et al. Middle meningeal artery embolization for chronic subdural hematoma: a series of 60 cases. Neurosurgery. 2019;85(6):801-807.

24. Ban SP, Hwang G, Byoun HS, et al. Middle meningeal artery embolization for chronic subdural hematoma. Radiology. 2018;286(3):992-999.

25. Haldrup M, Ketharanathan B, Debrabant B, et al. Embolization of the middle meningeal artery in patients with chronic subdural hematoma-a systematic review and meta-analysis. Acta Neurochir (Wien). 2020;162(4):777-784.

26. Waqas M, Vakhari K, Weimer PV, et al. Safety and effectiveness of embolization for chronic subdural hematoma: systematic review and case series. World Neurosurg. 2019;126: 228-236.

27. Court J, Touchette CJ, Iorio-Morin C, et al. Embolization of the middle meningeal artery in chronic subdural hematoma-a systematic review. Clin Neurol Neurosurg. 2019;186: 105464.

28. De Bonis P, Olei S, Mongardi L, et al. Chronic subdural hematoma in patients aged 80 years and older: a two-centre study. Clin Neurol Neurosurg. 2018;170:88-92.

29. Bartek J Jr, Sjåvik K, Ståhl F, et al. Surgery for chronic subdural hematoma in nonagenarians: a Scandinavian population-based multicenter study. Acta Neurol Scand. 2017; 136(5):516-520.

30. Gelabert-González M, Román-Pena P, Arán-Echabe E. Chronic subdural hematoma in the oldest-old population. Neurosurg Rev. 2018;41(4):983-984.

31. Christopher E, Poon MTC, Glancz LJ, et al. Outcomes following surgery in subgroups of comatose and very elderly patients with chronic subdural hematoma. Neurosurg Rev. 2019;42(2):427-431.

32. Krupa M, Stachura K, Moskala M. A comparison of the results of treatment in young and elderly patients with chronic subdural hematoma depending on the method of surgery. Article in Polish. Ann Acad Med Stetin. 2009;55(2):39-46.

33. Watanabe S, Kato N, Sato M, et al. Treatment outcomes of burr-hole surgery for chronic subdural hematoma in the elderly living beyond life expectancy: a study comparing cure, recurrence, and complications in patients aged $\geq 80$ years versus $\leq 79$ years. World Neurosurg. 2019;132:e812-e819.

34. Borger V, Vatter H, Oszvald Á, et al. Chronic subdural haematoma in elderly patients: a retrospective analysis of 322 patients between the ages of 65-94 years. Acta Neurochir (Wien). 2012;154(9):1549-1554.
35. Rohde V, Graf G, Hassler W. Complications of burr-hole craniostomy and closed-system drainage for chronic subdural hematomas: a retrospective analysis of 376 patients. Neurosurg Rev. 2002;25(1-2):89-94.

36. Knopman J, Link TW, Navi BB, et al. Rates of repeated operation for isolated subdural hematoma among older adults. JAMA Netw Open. 2018;1(6):e183737.

37. Ferrante G, Rao SV, Jüni P, et al. Radial versus femoral access for coronary interventions across the entire spectrum of patients with coronary artery disease: a meta-analysis of randomized trials. JACC Cardiovasc Interv. 2016;9(14): 1419-1434.

38. Zussman BM, Tonetti DA, Stone J, et al. Maturing institutional experience with the transradial approach for diagnostic cerebral arteriography: overcoming the learning curve. $J$ Neurointerv Surg. 2019;11(12):1235-1238.

\section{Disclosures}

Dr. Thomas: data safety monitoring board of SCENT trial, funds paid to institution by Stryker. Dr. Riina: consultant for Medtronic and speakers bureau for Stryker. Dr. Levy: ownership in NeXtGen Biologics, RAPID Medical, Claret Medical, Cognition Medical, Imperative Care, Rebound Therapeutics, StimMed, and Three Rivers Medical; consultant for Claret Medical, GLG Consulting, Guidepoint Global, Imperative Care, Medtronic, Rebound, and StimMed; honoraria from Medtronic; advisory board of Stryker, MEDX, Cognition Medical, and EndoStream Medical; and provider of medical/legal opinion as an expert witness. Dr. Spiotta: consultant for Penumbra, Terumo, and Stryker; and support of non-study-related clinical or research effort from Penumbra and Stryker. Dr. Gross: consultant for Medtronic and MicroVention. Dr. Khalessi: consultant for Medtronic and Cerenovus. Dr. Hanel: consultant for Medtronic. Dr. Levitt: consultant for Medtronic; ownership in Synchron, Cerebrotech, eLoupes, and Metis Innovative; and support of non-study-related clinical or research effort from Medtronic, Stryker, and Philips Volcano. Dr. Taussky: consultant for Covidien, Medtronic, and Stryker Neurovascular. Dr. Grandhi: consultant for Medtronic Neurovascular, Balt Neurovascular, Medtronic, and Cerenovus.

\section{Author Contributions}

Conception and design: Grandhi, Joyce, Bounajem, Taussky, Kan. Acquisition of data: Grandhi, Joyce, Thomas, Ogilvy, Riina, Tanweer, Levy, Spiotta, Gross, Jankowitz, Cawley, Khalessi, Pandey, Ringer, Hanel, Ortiz, Langer, Levitt, Binning, Kan. Analysis and interpretation of data: Grandhi, Joyce, Bounajem, Scoville. Drafting the article: Grandhi, Joyce, Bounajem, Taussky, Kan. Critically revising the article: Grandhi, Joyce, Bounajem, Thomas, Ogilvy, Riina, Tanweer, Levy, Spiotta, Gross, Jankowitz, Cawley, Khalessi, Pandey, Ringer, Hanel, Ortiz, Langer, Levitt, Taussky, Kan. Reviewed submitted version of manuscript: Grandhi, Joyce, Bounajem, Binning. Approved the final version of the manuscript on behalf of all authors: Grandhi. Statistical analysis: Scoville.

\section{Supplemental Information \\ Videos}

Video Abstract. https://vimeo.com/457720529.

\section{Correspondence}

Ramesh Grandhi: University of Utah, Salt Lake City, UT. neuropub@hsc.utah.edu. 